Research article

\title{
Distinct behavioral response of primary motor cortex stimulation in itch and pain after burn injury
}

\author{
Aurore Thibaut ${ }^{\mathrm{a}, \mathrm{b}, 1}$, Emily A. Ohrtman ${ }^{\mathrm{a}, 1}$, Leon Morales-Quezada ${ }^{\mathrm{a}}$, Laura C. Simko ${ }^{\mathrm{a}}$, \\ Colleen M. Ryan $^{\mathrm{d}}$, Ross Zafonte ${ }^{\mathrm{a}, \mathrm{c}}$, Jeffrey C. Schneider ${ }^{\mathrm{a}, 2}$, Felipe Fregni ${ }^{\mathrm{a}, *, 2}$ \\ ${ }^{a}$ Spaulding Rehabilitation Hospital, Harvard Medical School, Boston, MA, United States \\ b GIGA-Institute and Neurology Department, University of Liège and University hospital of Liège, Liège, Belgium \\ ${ }^{c}$ Massachusetts General Hospital and Brigham and Womens Hospital, Boston, United States \\ ${ }^{\mathrm{d}}$ Massachusetts General Hospital, Harvard Medical School, Boston, MA, Shriners Hospitals for Children-Boston, Boston, MA, United States
}

\section{A R T I C L E I N F O}

\section{Keywords:}

Burn

Pain

Itch

Neuromodulation

Transcranial direct current stimulation

\begin{abstract}
A B S T R A C T
It is still unclear whether chronic neuropathic pain and itch share similar neural mechanisms. They are two of the most commonly reported challenges following a burn injury and can be some of the most difficult to treat. Transcranial direct current stimulation (tDCS) has previously been studied as a method to modulate pain related neural circuits. Therefore, we aimed to test the effects of tDCS on post-burn neuropathic pain and itch as to understand whether this would induce a simultaneous modulation of these two sensory manifestations. We conducted a pilot randomized controlled clinical trial comprised of two phases of active or sham M1 tDCS (Phase I: 10 sessions followed by a follow-up period of 8 weeks; Phase II: additional 5 sessions followed by a follow-up period of 8 weeks, and a final visit 12 months from baseline). Pain levels were assessed with the Brief Pain Inventory (BPI) and levels of itch severity were assessed with the Visual Analogue Scale (VAS). Measurements were collected at baseline, after the stimulation periods, at 2, 4 and 8-week follow up both for Phase I and II, and at the final visit. Sixteen patients were assigned to the active group and 15 to the sham group. Ten sessions of active tDCS did not reduce the level of pain or itch. We identified that itch levels were reduced at 2-week followup after the sham tDCS session, while no placebo effect was found for the active group. No difference between active and sham groups was observed for pain. We did not find any treatment effects during Phase II. Based on these findings, it seems that an important placebo effect occurred during sham tDCS for itch, while active M1 tDCS seems to disrupt sensory compensatory mechanisms. We hypothesize that pain and itch are complementary but distinct mechanisms of adaptation after peripheral sensory injury following a burn injury and need to be treated differently.
\end{abstract}

\section{Introduction}

Within the last four decades, there have been notable advances in acute burn care and, subsequently, a decline in burn related mortality rates. As a result, there is a growing need to focus research on long term physical recovery and to develop effective post-acute treatments [1]. Chronic neuropathic pain and itch are two of the most commonly reported challenges following injury and can be some of the most difficult to treat. In a survey of 358 post-acute burn survivors, $52 \%$ reported having ongoing burn-related pain [2]. Similarly, in a study of 104 burn survivors, $28 \%$ experienced persistent symptoms of pain for 28 months or longer [3].

In addition to pain, itch is a common complaint among burn survivors. In a sample of 510 adult burn survivors, prevalence rates were as high as $87 \%$ [4]. Another study found that as many as $96 \%$ experienced three or more episodes a day and, of those burn survivors, $94 \%$ rated their itch as 'unbearable'(5). Previous research shows that chronic pain and itch can be significant causes of disability following injury and can limit patients' daily activities as well as affect quality of life [6]. However, despite its significance and prevalence, studies exploring chronic pain and itch, as well as effective treatment options, remain limited $[6,7]$.

\footnotetext{
* Corresponding author at: Spaulding Neuromodulation Centre, Spaulding Rehabilitation Hospital, Harvard Medical School, 79/96 13th Street, Charlestown, MA, United States.

E-mail addresses: athibaut@neuromodulationlab.org (A. Thibaut), Fregni.Felipe@mgh.harvard.edu (F. Fregni).

${ }^{1}$ Both authors have contributed equally to this work.

2 These two authors share senior authorship.
} 
Recent work on Transcranial Direct Current Stimulation (tDCS; i.e., direct constant current) has shown the efficacy of this noninvasive brain stimulation (NIBS) technique to modulate cortical activity in both healthy subjects and subjects with neurological conditions [8]. From a neurophysiological perspective, tDCS increases the neuronal excitability by facilitating the action potential release and the modification of NMDA receptors' excitability [9]. Moreover, tDCS may strengthen task-related dynamical synaptic connections [10]. This technique is widely used to modulate brain activity in research and in clinic for stroke rehabilitation [11], pain [12], depression [13], and tinnitus [14]. In healthy subjects, tDCS has been shown to modulate pain threshold and Conditioned Pain Modulation (CPM) [15]. A pilot study evaluating the effects of tDCS on pain in subjects with burn injuries showed that a single session of active anodal tDCS over the primary motor cortex may lead to a decrease in cortical excitability when compared to sham stimulation [16].

Based on the notion that burn injury leads to central neural changes associated with sensory deafferentation that results in itch $[17,18]$ and chronic pain, we hypothesized that the anodal tDCS-induced motor cortex excitability increase would enhance thalamic activity [15,17-19] and thus decrease maladaptive changes associated with sensory deafferentation leading to itch and pain. In this study, we aimed to assess the effects of tDCS on post-burn neuropathic pain and itch, as measured by the modified Brief Pain Inventory (BPI) and Visual Analog Scale (VAS), respectively.

\section{Methods}

\subsection{Participants}

Thirty-four subjects with burn injuries were enrolled in the study, and 31 subjects were randomized. All subjects were above the age of 18 , and rated their pain and/or itch as moderate or severe ( $\geq 4$ on the Visual Analogue Scale) within the last 3 weeks. Subjects had to be discharged from acute care and have burn injuries occurring at least 3 weeks prior to study enrollment. If subjects were taking Carbamazepine (Tegretol), Oxcarbaepine (Trileptil), or Phenytoin (Dilantin), they were excluded from the study. Medication history was taken at the time of consent and any changes in medication were recorded. Subjects were recruited through collaborating physicians in inpatient rehabilitation and outpatient settings. In addition, flyers were posted online and in outpatient burn clinics. Potential participants were contacted using the Partners Research Patient Data Registry (RPDR). Written informed consent was obtained from all patients according to the declaration of Helsinki. The study was approved by the Institutional Review Board at Spaulding Rehabilitation Hospital, Boston, MA. As the study comprises two phases, participants were asked to provide consent at the start of each phase to promote adherence.

\subsection{Study design}

This pilot study was a parallel double-blinded, placebo-controlled randomized trial. In total, the trial was comprised of 23 visits scheduled on weekdays. Visits were split into two phases to promote adherence.

During the baseline visit (Visit 1), subjects completed an EEG and scaled assessments (detailed below). Phase I was comprised of 10 stimulation visits over the course of 2 weeks (Visits 2-11) and 3 follow-up visits (Visits 12-14). In Phase II, subjects participated in 5 stimulation visits over 1 week (Visits 15-19), 3 follow-up visits (Visits 20-22), and a final visit (V23) approximately 12 months from baseline.

\subsection{Procedure}

Direct current was transferred by a saline-soaked pair of surface sponge electrodes $(35 \mathrm{~cm}$ [2]) and delivered by a battery-driven, constant current stimulator (Soterix Medical, New-York). The anodal electrode was placed over the primary motor cortex (M1), contralateral to the most painful/itchy side, and the cathode was placed over the opposite supra-orbital area. The duration of stimulation was $20 \mathrm{~min}$ at $2 \mathrm{~mA}$ with a ramp-up and ramp-down of $30 \mathrm{~s}$, as previously described [20]. For the sham condition, the stimulation was applied for $30 \mathrm{~s}$ with the same ramp-up and down, to mimic the active condition. The device we used allows the delivery of both active and sham stimulation, and all stimulation sessions were performed by a researcher who was not involved in any of the assessments, allowing for a double-blinded procedure.

\subsection{Assessments}

We used the Brief Pain Inventory (BPI) to assess the magnitude of pain reduction following active or sham tDCS [21]. The BPI is a short self-assessment questionnaire that provides information on various dimensions of pain including how pain developed, the types of pain a patient experiences, the time of day pain is experienced, as well as current ways of alleviating pain. The BPI also consists of the VAS pain scale, a 10 - point scale $(0=$ "no pain", $10=$ "pain at its worst") measuring a patient's worst and least pain, on average and at present time. The BPI provides information on the intensity of pain (the sensory dimension) as well as the degree to which pain interferes with function (the reactive dimension).

In addition to the BPI, we used the VAS itch scale, to measure itching severity and activity on average and at present time. This scale has previously been used in a study investigating treatment of refractory itching in burn patients [5]. The same scale (VAS anxiety scale) was used to evaluate anxiety. Subjects were instructed to keep a record of itching/pain activity and severity using this scale in a diary through Visit 12 . In addition, any medication changes were recorded.

A short, 9-question questionnaire was used to assess the side effects of tDCS. Following stimulation, participants were asked if they experienced any of the following symptoms: headache, neck pain, scalp pain, scalp burn, acute mood change, tingling, skin redness, sleepiness, trouble concentrating as well as any other unspecified side effect. If a participant experienced any of these side effects, they were asked to rate the severity on a 4-point scale ( $1=$ "Absent", $2=$ "Mild", $3=$ "Moderate", $4=$ "Severe"). In addition, they were asked to rate the likelihood that the symptom was related to tDCS on a 5-point scale $(1=$ "None", 2 = "Remote", 3 = "Possible", 4 = "Probable", $5=$ "Definite").

\subsection{Statistical analysis}

Differences in baseline values between the two stimulation conditions were analyzed with a Student $t$-test for continuous variables and with Chi-square tests for dichotomous variables.

All analyses were conducted according to the principle of intentionto-treat using a last observation carried forward for missing data.

For each outcome, a group analysis running a mixed ANOVA model was performed, in which the independent variables were time (baseline, post-stimulation, 2-week follow-up, 4-week follow-up and 8-week follow-up), condition of stimulation (sham vs. active), the interaction term (time vs. the condition), and subject ID. Cohen's effect sizes were also calculated for significant comparisons. To evaluate possible linear changes over time, we used a correlation between time and each variable of interest. As exploratory analysis, we also tested if the presence of pain ( 0 for no pain; 1 for presence of pain) modulated the effects of tDCS on itch (VAS itch changes after active or sham tDCS). Two-tailed p-values $<0.05$ were considered statistically significant in all cases. All statistical analyses were performed using STATA (StataCorp 2013. StataCorp LP, College Station, TX).

\section{Results}

Thirty-one patients were randomized to receive either active 


\section{Phase I}

A.

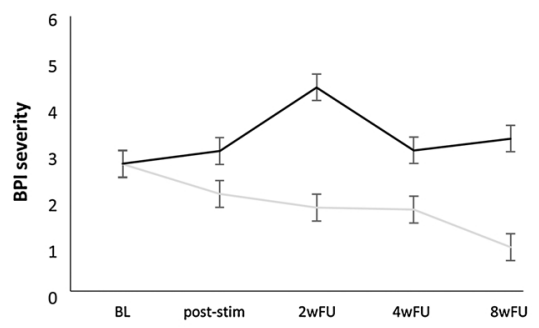

B.

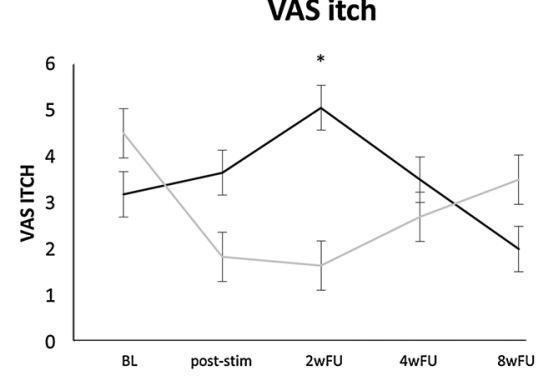

\section{Phase II}

A. BPI severity

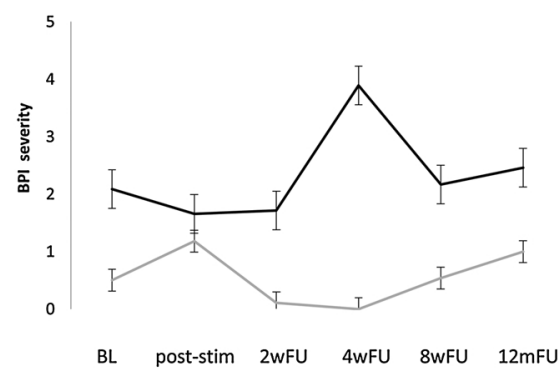

B.

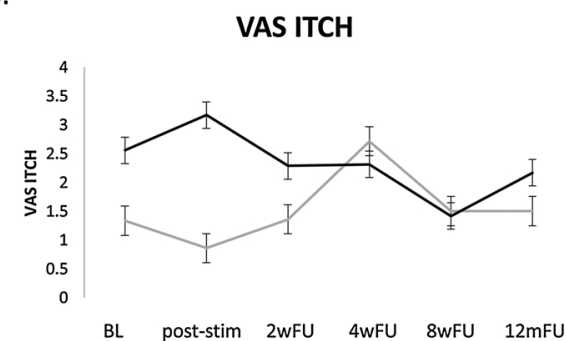

Table 1

Baseline characteristics of patients enrolled in the active and sham groups. * info missing for two subjects in the active group.

\begin{tabular}{|c|c|c|c|}
\hline & Active tDCS & Sham tDCS & $\begin{array}{l}\text { P values } \\
\text { (active/ } \\
\text { sham) }\end{array}$ \\
\hline Number of subjects & 16 & 15 & \\
\hline Age (years, mean $\pm S D$ ) & $49 \pm 14$ & $48 \pm 14$ & 0.99 \\
\hline $\begin{array}{l}\text { Gender (Number of males } \\
\quad-\%)\end{array}$ & $7(44 \%)$ & $8(53 \%)$ & 0.63 \\
\hline $\begin{array}{l}\text { Time since injury* (years, } \\
\text { mean } \pm S D \text { ) }\end{array}$ & $7.2 \pm 7.3$ & $11.5 \pm 10.5$ & 0.39 \\
\hline \multirow[t]{4}{*}{ Ethnicity } & 10 Caucasian & 9 Caucasian & 0.56 \\
\hline & 5 African & 5 African & \\
\hline & American & American & \\
\hline & 1 Hispanic & 1 Hispanic & \\
\hline $\begin{array}{l}\text { BDI at baseline } \\
\qquad(\text { mean } \pm \mathrm{SD})\end{array}$ & $11.2 \pm 9.1$ & $12.6 \pm 10.2$ & 0.70 \\
\hline $\begin{array}{l}\text { VAS anxiety at baseline } \\
\quad(\text { mean } \pm \text { SD) }\end{array}$ & $2.6 \pm 2.8$ & $2.1 \pm 2.0$ & 0.54 \\
\hline
\end{tabular}

$(n=16)$ or sham tDCS $(n=15)$. A flow chart illustrating the number of subjects who participated in each phase is presented in Fig. 1. Twentyone patients completed Phase I and were enrolled in Phase II. Fourteen completed all study visits (Phase I and II). Ten patients did not have any pain (5 in the active group and 5 in the sham group) at baseline and therefore, were not included in the analyses for the BPI. Eight patients did not complete the itch questionnaire (4 in the active group and 4 in the sham group) at baseline and were not included in the VAS itch analysis. Baseline comparisons between stimulation conditions showed no significant a priori differences (Table 1 ).

\subsection{Phase I}

The ANOVA showed no significant group*time interaction for BPI severity $(\mathrm{F}=0.55 ; \mathrm{p}=0.703)$.

The ANOVA showed a significant group*time interaction for itch severity at present time $(F=3.28 ; p=0.017$ - see Fig. 2). Post-hoc tests showed a significant difference between active and sham groups at 2-week follow-up ( $\mathrm{t}=-2.399 ; \mathrm{p}=0.02$; ES:1.15). The group that received active stimulation had worse itching scores than the group that received sham stimulation as there was a placebo effect (itching improvement) only in the sham group. No correlation with time was found.

No group*time interaction was observed for VAS anxiety $(\mathrm{F}=0.75$; $\mathrm{p}=0.661$ ). Detailed information for BPI, VAS itch, and VAS anxiety can be found in Table 2.

When looking at the correlation between variables, we did not find any correlation between BPI at baseline and VAS itch $(p=0.083)$ nor VAS anxiety $(\mathrm{p}=0.847)$.

\subsection{Phase II}

When comparing characteristics (i.e., age, time since injury, side of stimulation, gender) and treatment allocation (active vs. sham) of patients enrolled in Phase I with patients enrolled in Phase II, no significant differences were found.

We did not find any differences between active and sham groups for any of the outcome variables (see supplementary material).

The analysis testing whether the presence of pain was not found to mediate the effects of tDCS on itch $(p=0.567)$, thus showing that presence of pain does not influence the effects of tDCS on itch.

\subsection{Safety}

All study participants tolerated tDCS sessions well. The majority reported a mild-to-moderate tingling or itching sensation during both active and sham stimulations. No unexpected adverse effects were observed.

\section{Discussion}

In this randomized controlled clinical trial, we found that 10 sessions of tDCS did not reduce the level of pain or itch in burn injury patients. However, itch levels were reduced at 2-week follow-up after the sham tDCS session, indicating a possible placebo effect. On the other hand, the group who received active tDCS had no placebo effect, 


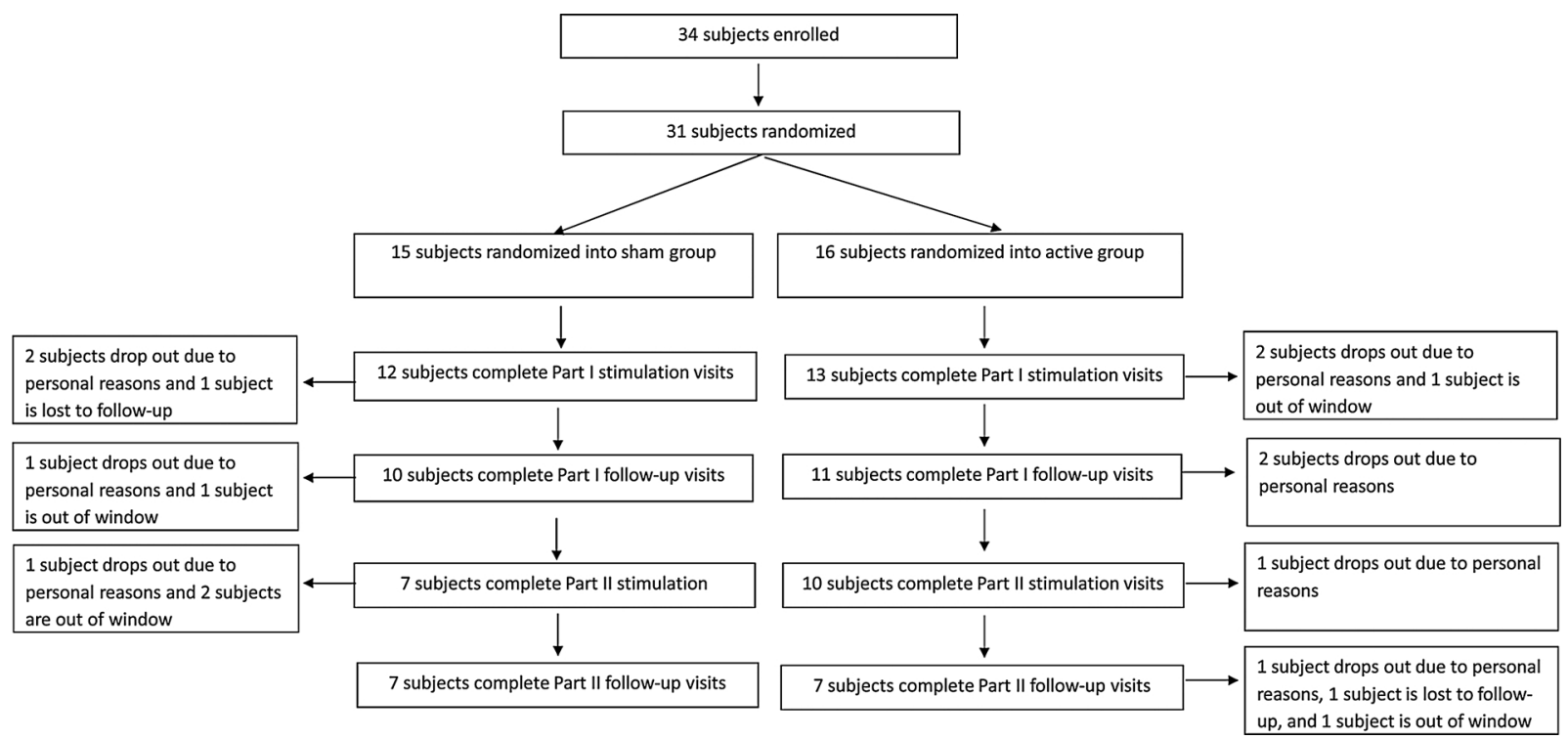

Fig. 2. Mean and standard error of brief pain inventory (BPI) total scores (A.) and the visual analogue scale (VAS) for itch (B.) at each time point for the active group (in black) and the sham group (in grey). The ANOVA revealed a significant effect of group*time interaction for VAS itch but not for BPI. * stands for significant difference between active and sham group at the 2-week follow-up. (For interpretation of the references to colour in this figure legend, the reader is referred to the web version of this article).

making it possible that tDCS counteracts this effect by blocking the subjective perception of itching in these subjects. For patients who continued to the second phase of this study, no difference between active and sham groups was found.

\subsection{Placebo or active nocebo effects}

Placebo is a robust psychological and neuro-physiological phenomenon that appears to be dependent largely on expectation [22]. This phenomenon is frequently observed in various pathologies including in patients suffering from chronic pain syndromes [23,24], with a positive impact on clinical outcomes [25].

Interestingly, in the present study, the sham condition seemed to induce an effect on itch reduction level. Placebo effect has already been observed after electrical stimulation [26]. The placebo effect could partially explain our findings following the sham stimulation. Indeed, positive expectations of the intervention (here, tDCS) have been shown to decrease anxiety in patients with severe burn injuries [27]. This decrease in anxiety has an impact on both pain and itch; moreover, symptom conscientiousness modifies the way patients report their symptoms on clinical scales, which seems to be related to specific genetic variations [28]. On the other hand, it seems that the active tDCS blocked this placebo effect. One possibility to explain these effects is that tDCS may have worsened itching and thus blocked potential positive effects of placebo.

\section{2. tDCS over the motor cortex increases sensory threshold}

As tDCS increases sensory threshold [15], it may induce increased itch levels in burn patients, or, as observed here, may block or revert the placebo effect. One could hypothesize that decreased deafferentation may lead to compensatory changes, and itching may be a peripheral compensatory mechanism to increase peripheral sensory afference. In this scenario, tDCS central modulation may decrease response to peripheral afference thus blocking the potential placebo effect of itching regulation [31,32]. In addition, this increase in itch sensation, may have annihilated tDCS effects on pain, since itch and pain mechanisms are linked.

Therefore, another target area can modulate the expected tDCS-related analgesic effects. Indeed, considering that the prefrontal areas are involved in downregulation of affective conditions such as pain, it is possible that tDCS delivered over dorsolateral prefrontal cortex is more relevant for modulation of the emotional experience of pain than for the somatosensory aspect, operationalized pain intensity. However, further studies are needed to clarify this point.

Another possibility would be to test different parameters of stimulation. It has been shown that by modulating some tDCS parameters such as duration, time, or polarity of stimulation, reversal effects could be observed [33,34].

\subsection{The lack of significant effects of tDCS in pain}

The lack of analgesic effect of M1 tDCS could be related to low

Table 2

Phase I results for BPI, VAS itch and VAS anxiety presented in mean and SD at each time points for active and sham groups. * stands for a significant difference between the two groups.

\begin{tabular}{|c|c|c|c|c|c|c|c|c|c|}
\hline & \multicolumn{3}{|l|}{ BPI severity } & \multicolumn{3}{|l|}{ VAS itch } & \multicolumn{3}{|l|}{ VAS anxiety } \\
\hline & ACTIVE & SHAM & $\mathrm{p}$-value & ACTIVE & SHAM & $\mathrm{p}$-value & ACTIVE & SHAM & $\mathrm{p}$-value \\
\hline Baseline & $3.59(1.52)$ & $4.68(1.89)$ & 0.26 & $3.18(2.78)$ & $4.50(3.15)$ & 0.30 & $2.60(2.32)$ & $2.07(2.34)$ & 0.54 \\
\hline After stimulation & $4.45(1.94)$ & $4.19(1.45)$ & 0.76 & $3.65(2.91)$ & $1.83(1.76)$ & 0.09 & $3.25(3.62)$ & $2.92(3.37)$ & 0.82 \\
\hline 2-week follow-up & $4.81(2.08)$ & $3.89(2.04)$ & 0.39 & $5.05(3.52)$ & $1.65(2.29)$ & $0.02 *$ & $2.91(2.56)$ & 1.21 (1.97) & 0.09 \\
\hline 4-week follow-up & $4.41(2.02)$ & $4.25(2.26)$ & 0.90 & $3.50(2.15)$ & $2.70(2.37)$ & 0.45 & $2.44(2.24)$ & $2.82(3.28)$ & 0.77 \\
\hline 8-week follow-up & $4.21(2.27)$ & $3.31(1.90)$ & 0.50 & $2.00(2.00)$ & $3.50(3.04)$ & 0.37 & $3.06(3.17)$ & $1.31(1.58)$ & 0.17 \\
\hline
\end{tabular}


levels of pain and increased itching that may be a compensatory mechanism for pain modulation. However, it should be noted that, in the present study, we did not find a significant correlation between pain and itch levels $(p=0.083)$. This could be explained by the small sample size.

By definition, neuropathic pain occurs as a result of lesions or disease to the somatosensory nervous system [35]. Given the extensive damage to the somatosensory system following burn injury, patients can develop neuropathic pain. Many of the signs and symptoms in patients with burn injuries resemble features that are often present in those with neuropathic pain $[6,36]$. One symptom of neuropathic pain is central sensitization, which appears to be a key contributor to burninduced pain. Chronic, bilateral hyperexcitability of wide dynamic range neurons, and microglial activation in the spinal cord often occurs after burn injuries [37]. In addition, after a burn injury, many patients demonstrate reduced thermal pain thresholds in regions outside the burn injury area [38]. Further research is needed to determine how patients with moderate to severe pain due to burn would respond to tDCS of motor cortex.

Therefore, our results favor the notion of different neural circuitry modulating itch and pain related central sensitization, as previously suggested [39-42]. However, here, we show that these distinct pathways have a clinical impact on treatment efficacy.

\subsection{Limitations}

Several limitations of this study must be considered. First, the sample size is relatively small, and the baseline characteristics of the patients were heterogonous in terms of level of pain, severity of itch, and duration of the symptoms. It can be hypothesized that M1 tDCS could be helpful for a subgroup of patients; however, given the limited number of subjects included in the study, subgroup analysis could not be performed. Another important limitation is the rate of drop-out. Indeed, almost a third of the subjects dropped-out after the two weeks of stimulations. This could have impacted the results, even if we did not find any differences in term of rate of drop-out between the active and the sham groups. A home-based remotely supervised intervention may overcome this challenge.

\section{Conclusion}

A placebo effect was observed during sham tDCS, while active tDCS applied to the primary motor cortex seems to disrupt sensory compensatory mechanisms. Therefore, M1 tDCS may not be a suitable treatment to treat pain and itch in patients with a burn injury. Given these results, we hypothesize that pain and itching are complementary but distinct mechanisms of adaptation after peripheral sensory injury following a burn. Based on the present findings and related hypothesis, pain and itch should be treated in different ways. Therefore, when designing a clinical trial, a specific approach should be developed to reduce itch rather than merging itch and pain together.

\section{Source of funding}

The contents of this manuscript were developed under a grant from the National Institute on Disability, Independent Living, and Rehabilitation Research, NIDILRR grant number 90DP0035. NIDILRR is a Center within the Administration for Community Living (ACL), Department of Health and Human Services (HHS). The contents of this manuscript do not necessarily represent the policy of NIDILRR, ACL, HHS, and you should not assume endorsement by the Federal Government

\section{Acknowledgments}

Dr. Aurore Thibaut is a FNRS is a post-doctoral research fellow. Dr.
Morales-Quezada received funding support from an Institutional National Research Service Award from the National Center for Complementary and Integrative Health grant T32AT000051, the Ryoichi Sasakawa Fellowship Fund, and the Program in Placebo Studies at Beth Israel Deaconess Medical Center. The contents of this manuscript were developed under a grant from the National Institute on Disability, Independent Living, and Rehabilitation Research, NIDILRR grant numbers 90DP0035, 90DPBU0001, and 90DP0055. NIDILRR is a Center within the Administration for Community Living (ACL), Department of Health and Human Services (HHS). The contents of this manuscript do not necessarily represent the policy of NIDILRR, ACL, HHS, and you should not assume endorsement by the Federal Government.

\section{References}

[1] C.M. Ryan, D.A. Schoenfeld, W.P. Thorpe, R.L. Sheridan, E.H. Cassem, R.G. Tompkins, Objective estimates of the probability of death from burn injuries, N. Engl. J. Med. 338 (6) (1998) 362-366, https://doi.org/10.1056/ NEJM199802053380604 [Internet] Available from:.

[2] A. Dauber, P.F. Osgood, A.J. Breslau, H.L. Vernon, D.B. Carr, Chronic persistent pain after severe burns: a survey of 358 burn survivors, Pain Med. 3 (1) (2002) 6-17.

[3] R. Egyhazi, F. Fregni, G.L. Bravo, N.H.T. Trinh, C.M. Ryan, J.C. Schneider, Chronic pain following physical and emotional trauma: the station nightclub fire, Front Neurol. 5 (June) (2014).

[4] N.E.E. Van Loey, M. Bremer, A.W. Faber, E. Middelkoop, M.K. Nieuwenhuis, Itching following burns: epidemiology and predictors, Br. J. Dermatol. 158 (1) (2008) 95-100.

[5] L.K.S. Parnell, B. Nedelec, G. Rachelska, L. Lasalle, Assessment of pruritus characteristics and impact on burn survivors, J. Burn Care Res. 33 (3) (2012) 407-418.

[6] J.C. Schneider, N.L. Harris, Shami A. El, R.L. Sheridan, J.T. Schulz, M.L. Bilodeau, et al., A descriptive review of neuropathic-like pain after burn injury, J. Burn Care Res. 27 (4) (2006) 524-528.

[7] C. Richardson, D. Upton, M. Rippon, Treatment for wound pruritus following burns, J. Wound Care 23 (5) (2014) 227-233, https://doi.org/10.12968/jowc.2014.23.5. 227 [Internet].Available from:

[8] M.A. Nitsche, L.G. Cohen, E.M. Wassermann, A. Priori, N. Lang, A. Antal, et al., Transcranial direct current stimulation: state of the art 2008, Brain Stimul. 1 (3) (2008) 206-223 [Internet]. 2008/07/01. Available from: http://www.ncbi.nlm. nih.gov/pubmed/20633386.

[9] C.J. Stagg, M.A. Nitsche, Physiological basis of transcranial direct current stimulation, Neuroscientist 17 (1) (2011) 37-53 [Internet].Available from: http://www. ncbi.nlm.nih.gov/pubmed/21343407.

[10] R. Polania, W. Paulus, M.A. Nitsche, Modulating cortico-striatal and thalamo-cortical functional connectivity with transcranial direct current stimulation, Hum. Brain Mapp. 33 (10) (2012) 2499-2508 [Internet]. Available from: http://www. ncbi.nlm.nih.gov/pubmed/21922602.

[11] J. Marquez, P. van Vliet, P. McElduff, J. Lagopoulos, M. Parsons, Transcranial direct current stimulation (tDCS): does it have merit in stroke rehabilitation? A systematic review, Int. J. Stroke 10 (April (3)) (2015) 306-316.

[12] M.F. DosSantos, N. Ferreira, R.L. Toback, A.C. Carvalho, A.F. DaSilva, Potential Mechanisms Supporting the Value of motor cortex Stimulation to Treat Chronic Pain Syndromes Vol. 10 Frontiers in Neuroscience, 2016.

[13] U. Palm, A. Hasan, W. Strube, F. Padberg, tDCS for the treatment of depression: a comprehensive review, Eur. Arch. Psychiatry Clin. Neurosci. (2016) 1-14.

[14] G.S. Shekhawat, C.M. Stinear, G.D. Searchfield, Modulation of perception or emotion? A scoping review of tinnitus neuromodulation using transcranial direct current stimulation, Neurorehabil. Neural Repair (2015) [Internet]. 1545968314567152-. Available from: http://nnr.sagepub.com/content/early/ 2015/02/10/1545968314567152.abstract.

[15] J.S. Reidler, M.E. Mendonca, M.B. Santana, X. Wang, R. Lenkinski, A.F. Motta, et al., Effects of motor cortex modulation and descending inhibitory systems on pain thresholds in healthy subjects, J. Pain 13 (5) (2012) 450-458.

[16] A. Portilla, G. Bravo, F. Miraval, M. Villamar, J. Schneider, C. Ryan, et al., A feasibility study assessing cortical plasticity in chronic neuropathic pain following burn injury, J. Burn care Res. 34 (2013) e48-e52 [Internet] Available from: http:// cochranelibrary-wiley.com/o/cochrane/clcentral/articles/372/CN-00912372/ frame.html.

[17] M. Simis, J.S. Reidler, D. Duarte Macea, I. Moreno Duarte, X. Wang, R. Lenkinski, et al., Investigation of central nervous system dysfunction in chronic pelvic pain using magnetic resonance spectroscopy and noninvasive brain stimulation, Pain Pract. 15 (5) (2015) 423-432 [Internet] Available from: http://www.ncbi.nlm.nih gov/pubmed/24799153.

[18] A.F. Dasilva, M.E. Mendonca, S. Zaghi, M. Lopes, M.F. Dossantos, E.L. Spierings, et al., TDCS-induced analgesia and electrical fields in pain-related neural networks in chronic migraine, Headache 52 (8) (2012) 1283-1295.

[19] M.F. DosSantos, T.M. Love, I.K. Martikainen, T.D. Nascimento, F. Fregni, C. Cummiford, et al., Immediate effects of tDCS on the $\mu$-opioid system of a chronic pain patient, Front. Psychiatry 3 (November) (2012).

[20] F. Fregni, P.S. Boggio, M.C. Lima, M.J. Ferreira, T. Wagner, S.P. Rigonatti, et al., A 
sham-controlled, phase II trial of transcranial direct current stimulation for the treatment of central pain in traumatic spinal cord injury, Pain 122 (1-2) (2006) 197-209 [Internet]. 2006/03/28.Available from: http://www.ncbi.nlm.nih.gov/ pubmed/16564618.

[21] T.N. Bryce, C.N. Budh, D.D. Cardenas, M. Dijkers, E.R. Felix, N.B. Finnerup, et al., Pain after spinal cord injury: an evidence-based review for clinical practice and research. Report of the national institute on disability and rehabilitation research spinal cord injury measures meeting, J. Spinal Cord Med. (2007).

[22] L. Colloca, D. Finniss, Nocebo effects, patient-clinician communication, and therapeutic outcomes. Vol. 307, JAMA - J. Am. Med. Assoc. (2012) 567-568.

[23] A. Watson, A. Power, C. Brown, W. El-Deredy, A. Jones, Placebo analgesia: cognitive influences on therapeutic outcome, Arthritis Res. Ther. 14 (2012).

[24] P. Reicherts, A.B.M. Gerdes, P. Pauli, M.J. Wieser, Psychological placebo and nocebo effects on pain rely on expectation and previous experience, J. Pain 17 (2) (2016) 203-214.

[25] A.W.M. Evers, L. Colloca, C. Blease, M. Annoni, L.Y. Atlas, F. Benedetti, et al., Implications of placebo and nocebo effects for clinical practice: expert consensus, Psychother. Psychosom. (2018).

[26] F. Fregni, P.S. Boggio, F. Bermpohl, F. Maia, S.P. Rigonatti, E.R. Barbosa, et al., Immediate placebo effect in Parkinson's disease-is the subjective relief accompanied by objective improvement? Eur. Neurol. 56 (4) (2006) 222-229 [Internet]. 2006/10/24.Available from: http://www.ncbi.nlm.nih.gov/pubmed/17057382.

[27] M. Hosseini Amiri, S.H. Tavousi, S.R. Mazlom, Z.S. Manzari, Effect of transcranial direct current stimulation on pain anxiety during burn wound care, Burns 42 (4) (2016) 872-876.

[28] K.T. Hall, B.R. Tolkin, G.M. Chinn, I. Kirsch, J.M. Kelley, A.J. Lembo, et al., Conscientiousness is modified by genetic variation in catechol-O-methyltransferase to reduce symptom complaints in IBS patients, Brain Behav. 5 (1) (2015) 39-44.

[31] A. Flood, G. Waddington, S. Cathcart, High-definition transcranial direct current stimulation enhances conditioned pain modulation in healthy volunteers: a randomized trial, J. Pain (2016)

[32] P.S. Boggio, S. Zaghi, M. Lopes, F. Fregni, Modulatory effects of anodal transcranial direct current stimulation on perception and pain thresholds in healthy volunteers, Eur. J. Neurol. 15 (10) (2008) 1124-1130 [Internet]. 2008/08/23.Available from: http://www.ncbi.nlm.nih.gov/pubmed/18717717.

[33] C.J. Stagg, G. Jayaram, D. Pastor, Z.T. Kincses, P.M. Matthews, H. Johansen-Berg, Polarity and timing-dependent effects of transcranial direct current stimulation in explicit motor learning, Neuropsychologia 49 (2011) 800-804.

[34] K. Fricke, A.A. Seeber, N. Thirugnanasambandam, W. Paulus, M.A. Nitsche, J.C. Rothwell, Time course of the induction of homeostatic plasticity generated by repeated transcranial direct current stimulation of the human motor cortex, J Neurophysiol. 105 (2011) 1141-1149.

[35] S.P. Cohen, J. Mao, Neuropathic pain: mechanisms and their clinical implications, BMJ. 348 (February05 6) (2014) f7656-f7656.

[36] P. Gray, Acute neuropathic pain: diagnosis and treatment, Curr. Opin. Anaesthesiol. 21 (5) (2008) 590-595.

[37] L.M. Johanek, D.A. Simone, Activation of peripheral cannabinoid receptors attenuates cutaneous hyperalgesia produced by a heat injury, Pain 109 (3) (2004) 432-442.

[38] T.Z. Fischer, S.G. Waxman, Extraterritorial temperature pain threshold abnormalities in subjects with healed thermal injury, J. Rehabil. Res. Dev. 49 (4) (2012) 515.

[39] T. Liu, R.R. Ji, New insights into the mechanisms of itch: are pain and itch controlled by distinct mechanisms? Pflugers Arch. Eur. J. Physiol. (2013).

[40] L. Misery, E. Brenaut, R. Le Garrec, C. Abasq, S. Genestet, P. Marcorelles, et al., Neuropathic pruritus, Nat. Rev. Neurol. (2014).

[41] I. Goutos, Neuropathic mechanisms in the pathophysiology of burns pruritus: redefining directions for therapy and research, J. Burn Care Res. (2013).

[42] H.H. Andersen, A.I.M. van Laarhoven, J. Elberling, L. Arendt-Nielsen, Modulation of itch by conditioning itch and pain stimulation in healthy humans, J. Pain (2017). 